\title{
METHODOLOGY TO SUPPORT SEMANTIC RESOURCES INTEGRATION IN THE CONSTRUCTION SECTOR
}

\author{
Simona Barresi, Yacine Rezgui, Farid Meziane \\ School of Computing, Science and Engineering,University of Salford, Salford, U nited Kingdom \\ s.barresi@salford.ac.uk,y.rezgui@salford.ac.uk,f.meziane@salford.ac.uk \\ Celson Lima \\ CSTB, Sophia Antipolis, France \\ c.lima@cstb.fr.
}

Keywords: Ontology, Semantic Resources, Schema Matching.

\begin{abstract}
Ontologies, taxonomies, and other semantic resources, are used in a variety of sectors to facilitate knowledge reuse and information exchange between people and applications. In recent years, the need to access multiple semantic resources has led to the development of a variety of projects and tools aiming at integrating existing resources. This paper describes the methodology used during the FUNSIEC project, to develop an open infrastructure for the European Construction sector (OSIECS). This infrastructure aims towards facilitating integration among Construction related semantic resources, providing a base for the development of a new generation of e-services for the domain.
\end{abstract}

\section{INTRODUCTION}

Ontologies represent an important branch of traditional philosophy concerned with "the set of things whose existence is acknowledged by a particular theory or system of thought" (Guarino et al. 1999).

Various definitions of ontology have been proposed; the most quoted being the one formulated by Gruber (1994), who defined an ontology as a formal, explicit specification of a shared conceptualization. Therefore, an ontology defines the basic terms and relations that form the vocabulary of a topic area, as well as the rules for combining these terms and relations between terms (Neches et al. 1991).

Semantic integration of heterogeneous databases (Partridge 2002), content-based retrieval of yellow pages as well as product catalogues (Guarino et al 1999), and management of corporate memory (CoMMA 2000), are just some of the areas that have increasingly exploited the benefits deriving from the use of Semantic Resources (SRs) ${ }^{1}$ and ontology engineering in general.

\footnotetext{
${ }^{1}$ Semantic Resource is an expression coined in the SPICE project to refer to ontology-similar entities, such as dictionaries, taxonomies, etc.
}

Specifically in the construction sector, the need for improved communication and understanding between projects' stakeholders has led to an increased development of domain specific ontologies and SRs.

Construction is a knowledge intensive industry with its unique work settings and virtual organization like modus operandi (Rezgui 2001). This industry is heterogeneous and highly fragmented, consisting of numerous small and medium enterprises (SMEs) working together on various building projects. One of the major consequences is the difficulty in effective and efficient communication among partners during a building project, or between clients and suppliers of construction products. Several initiatives have tried to overcome this problem by developing a variety of SRs focused on Construction related terms. However, these initiatives tend to be country specific and not adapted to the multi-national nature of the sector. Also, these resources tend to be specialized for dedicated applications or engineering functions, e.g. product libraries.

In order to improve communication and information exchange between the various stakeholders during a construction project and to enable the development of a new generation of eservices for the sector, accessing a single semantic 
resource is no longer adequate. New initiatives, targeting the interoperability and integration of existing Construction related SRs are therefore needed.

This paper describes the methodology used to develop an Open Semantic Infrastructure for the Construction Sector (OSIECS). The purpose of OSIECS is to map Construction domain semantic resources between each other. This paper presents the research carried out within the FUNSIEC project. FUNSIEC was funded under the European eContent programme and aimed at evaluating the feasibility of building and maintaining OSIECS. The FUNSIEC consortium consisted of CSTB, the University of Salford, and UNINOVA.

\section{METHODOLOGIES AND TOOLS}

As reported in the literature (Corcho et al. 2003; Fernandez-Lopez 1999), various methodologies and tools have been developed in the field of ontology. Such as a variety of methodologies for building ontologies (Aussenac-Gilles 2001; Blazquez et al. 2001; Holsapple and Joshi 2002; Kayed and Colomb 2002; Pinto et al. 1999; Pinto and Martins 2000), methodologies for ontology reengineering (Klein 2001), methodologies for ontology learning (Kietz et al. 2000), methodologies for ontology evaluation (Gruninger and Fox 1995; Guarino and Welty 2000; Kalfoglou and Robertson 1999), methodologies for ontology evolution (Klein et al. 2002; Klein and Fensel 2001; Noy and Klein 2002), and methodologies and techniques for ontology mapping, merging, and alignment (Kalfoglou and Schorelmmer 2003; Noy and Musen 2000).

The establishment of a consensual and unified methodology is difficult, as is suggested by the existence of a variety of methodologies, each developed for a specific purpose. Possible reasons for these difficulties could be related to the lack of maturity of the field (Fernandez-Lopez 1999) or to the problems of adapting a unique methodology to a variety of different applications, sector and settings.

Environments supporting the development and management of ontologies through graphical interfaces have also proliferated, some providing tools for specific functions, such as integration and merging, or reason capabilities.

The latter category includes OntoEdit (Sure et al. 2002), OilEd (Bechhofer et al. 2001), Protégé (Grosso et al. 1999) - which support not only OIL, but also other models such as RDF_Ontolingua (Farquhar et al. 1996) and Ontosaurus (Lenat and Guha 1990). Furthermore, as described in (Noy and Musen 2000), the former category (environments supporting ontology merging) includes OntoMorph (Chalupsky 2000) Prompt (Noy and Musen 2000), and Chimaera (McGuiness et al. 2000). Other mapping and merging related techniques and tool reported in the literature include FCA-Merge (Stumme and Maedche 2001), Glue and IF-Map (Kalfoglou and Schorelmmer 2003).

In the context of FUNSIEC, schema matching represents a fundamental operation. In fact, a semantic infrastructure supporting integration must inevitably deal with the problems inherent to heterogeneous SRs, which may differ in both structure and terminology. Through schema matching two schemas are compared and the mapping between elements that correspond semantically to each other is produced $(\mathrm{Li}$ and Clifton 1994; Milo and Zohar 1998; Rahm and Bernstein 2001). However, schema matching is considered to be a time consuming and error prone process due to the fact that it is still predominantly performed manually. A comprehensive taxonomy, covering many of the existing approaches to automatic schema matching, is proposed by Rahm and Bernsteina (2001).

\section{SEMANTIC RESOURCES IN THE CONSTRUCTION SECTOR}

Among the multitude of SRs developed in the Construction sector, ranging from domain dictionaries to specialized taxonomies, some of the most notable efforts include the BS6100, bcXML, the ISO 12006-3, and the IFC (Industrial Foundation Classes).

The BS6100 (Glossary of Building and Civil Engineering terms), produced by the British Standards Institution, is a rich and complete glossary. It provides a comprehensive number of synonyms per term that can contribute towards any ontology development effort in the sector.

The bcXML (eConstruct 2001) is an XML vocabulary developed by the eConstruct IST project for the Construction industry. The bcXML provides the foundation for the development of the bcBuildingDefinitions taxonomy, which can be instantiated to create catalogue contents. Through bcXML, eConstruct has enabled the creation of 
"requirements messages" that can be interpreted by computer applications to then find suitable products and services that meet those requirements.

The ISO 12006-3 (ISO 2004) defines a schema for a generic taxonomy model, which enables the definition of concepts by means of properties, to group concepts, and to define relationships between concepts.

The IFC model, developed by the IAI (International Alliance for Interoperability), has produced a specification of data structures with the aim of supporting the development of the 'Building Information Model' where all the information about the whole life cycle of a construction project would be stored and shared among the actors involved.

All of the above resources, although different in terms of formalism, scope, details and applicability, can be used in a complementary manner. Providing an infrastructure to map these resources helps to overcome problems related to SRs' different formalism and inconsistencies, and enables effective reuse of existing Construction related SRs. This in turn facilitates the efficient use of knowledge within the sector and can support the implementation of eservices for the Construction domain.

\section{FUNSIEC METHODOLOGY}

As previously stated, numerous methodologies for ontology mapping, merging and alignment are reported in the literature. Determining the most appropriate methodology to be applied is dependent on the nature, individual characteristics, and applications of the domain in question. In the case of an open semantic infrastructure, such as OSIECS, the applied methodology has to satisfy the following requirements: (i) Make use of already established and recognized semantic resources. (ii) The infrastructure should be flexible and comprehensive enough to accommodate different business scenarios. (iii) The infrastructure is a living system and should allow for future expansion (including expansion of SRs or inclusion of new SRs). (iv) The end-user perspective and evaluation should be considered when planning expansion.

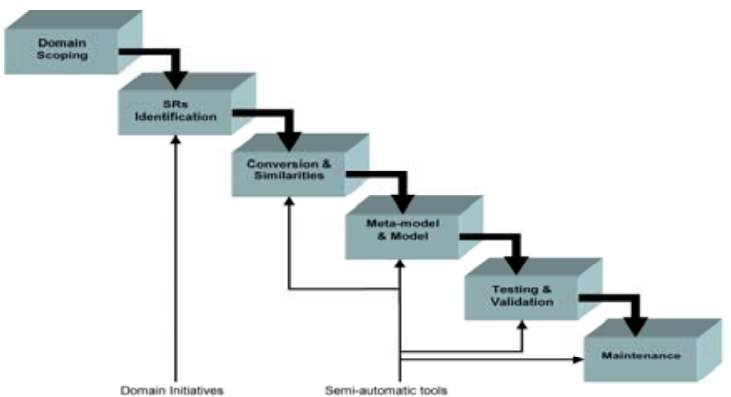

Figure 1. Phases of the FUNSIEC Methodology
Consequently, building upon the strength of numerous established methodologies, a new methodology was developed to guide the specification of the OSIECS infrastructure.

The FUNSIEC methodology (Figure 1) comprises of the following phases: domain scoping, candidate semantic resources identification, conversion and similarity detection (OSIECS Kernel), OSIECS meta-model and model construction, testing and validation, and maintenance.

The following sections provide a description of these stages. For pragmatic reasons, the conversion and similarity detection (OSIECS Kernel) and the OSIECS meta-model and model phases are here discussed as a single phase.

\subsection{Domain Scoping}

Scoping the domains (e.g. knowledge management, e-procurement, etc.) to be covered by OSIECS was facilitated by the use of typical scenarios that the infrastructure was expected to handle. The use of scenarios facilitates the description of the domain to be covered, how OSIECS was expected to be used, and which type of information it was expected to provide.

Two example scenarios are: (i) A designer developing a CAD drawing (IFC compliant) needs to also know the regulations to be followed in his/her project. In this case OSIECS would provide a link between the IFC tool and the e-COGNOS tool. (ii) An expert looking for information on the fire resistance of a given brick also needs to receive information on alternative products (suppliers, prices, etc.). OSIECS would then provide a link between the e-COGNOS tool and the e-Construct tool.

\subsection{Semantic Resources Identification}

The results of the first stage of the methodology aided the process of selecting the SRs to be included in OSIECS. For instance, knowledge management was recognized as one of the domains to be covered by the infrastructure, E-COGNOS was therefore included among the OSIECS components, because of its focus on construction concepts related to the consistent knowledge representation of (construction) knowledge items.

Existing Construction related SRs were selected for inclusion by considering their domain and a 
series of other features, such as their availability, cost, formalism, and underlining language. The SRs included in OSIECS are the e-Cognos ontology, the IFC model, the bcBuildingDefinition taxonomy, and the STABU LexiCon. The latter is a vocabulary of terms for the Construction industry and as such an implementation of ISO DIS 12006-3.

\subsection{Conversion and similarity detection - Meta-model and model construction}

After selecting the SRs to be included in OSIECS, syntax related problems (data heterogeneity) were addressed by converting each SRs' meta-schemas and schemas into the Web Ontology Language (OWL). This conversion facilitated the processes of dealing semi-automatically with semantic heterogeneity and detecting similarities between SRs' schemas. The conversion process produced the "rules of conversion" from each original formalism into OWL, which were used to create the OWL version of SRs' meta-schemas and schemas. At this stage, human intervention was required to identify the formalism used in SRs, study the semantics of the formalism, and identify syntactic elements in OWL corresponding to the syntactic elements of the formalism used in the SRs.

The next step in the construction of the OSIECS meta-model and model was to detect and validate the similarities existing among SRs' meta-schemas, and subsequently the ones existing among the different SRs' schemas. Two components were used for this purpose, a Detector of Mappings and a Validator. The Detector of Mappings used an inference engine (FONDIL ${ }^{2}$ ) to compare SRs' metaschemas and schemas and to create lists of equivalent or subsumed concepts. The Validator component was then used to check the similarities detected. The latter was a semi-automatic process, which required the intervention of human experts to ensure that the results of the validation process were correct and to add new similarities if required.

\subsection{Testing and Validation}

The testing and validation phase was directed at verifying the completeness of the infrastructure in terms of the conceptualization of targeted domains, assessing the relevance of concepts and

\footnotetext{
${ }^{2}$ FONDIL is available at http://195.83.41.67/ondil/connect.html
}

relationships, and verifying the consistency and coherency of concepts. To test and validate the OSIECS infrastructure a series of dedicated services and scenarios were implemented.

\subsection{Maintenance}

The final phase of the methodology is an ongoing process aimed at correcting and updating the open semantic infrastructure during its working life. Maintenance is required to eliminate errors or deficiencies in the infrastructure and to update and enrich the domains covered by OSIECS, through the integration of new SRs. In order to achieve this integration, new mappings and methods have to be considered

\section{THE OSIECS TRIAD}

By the use of the FUNSIEC methodology the OSIECS Triad was implemented, specifically the OSIECS Kernel, the OSIECS meta-model, and the OSIECS model. This section outlines the architecture of the OSIECS Kernel, a semiautomatic tool used to create both the OSIECS metamodel and model. As mentioned, the OSIECS Kernel covers two levels, the meta-schema and schema levels.

The Kernel consists of the following components: the Syntax Converter, the Semantic Analyser, the Converter, the Detector of Mappings, and the Validator. The operation of the OSIECS Kernel is depicted in figure 2. The role of the experts is to verify the results produced by the Syntactic Converter and the Semantic Analyser, as well as to help validate the lists produced by the FONDIL system.

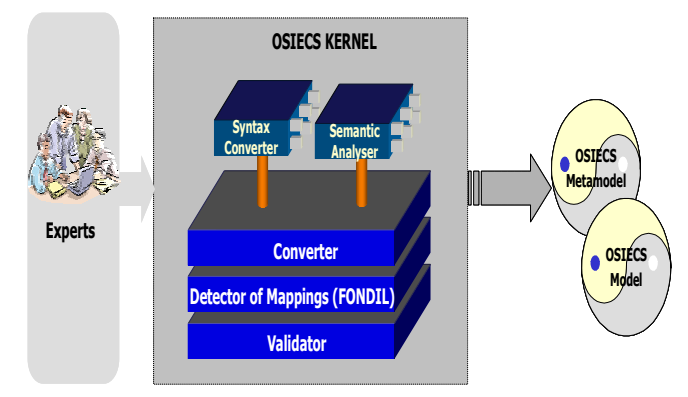

Figure 2. The OSIECS Kernel

The OSIECS meta-model and model are built by using the meta-schemas and schemas of the previously selected Construction related SRs. The 
Syntax converter and the Semantic analyser work together, using the meta-schemas and schemas of the four selected SRs as input, to produce the rules of conversion. These rules are then used by the Converter to guide the production of the OWL metaschemas and schemas for each of the SRs in the Kernel. The Detector of Mappings is played by the FONDIL system (briefly introduced below), which works with the OWL-converted metaschemas/schemas to produce a list of equivalent or subsumed entities. These entities are then analysed and assessed by the Validator (Lima et al. 2005c).

Helped by the appropriate software tools, the experts play an essential role in the creation of OSIECS meta-model/model. They participate at both levels, taking care of: (i) the manual analysis of the SRs and their respective meta-schemas/schemas; (ii) the analysis of the rules of conversion; (iii) the assessment of the detection of similarities; (iv) the inspection of the validation process; and (v) the assessment of the final output.

\subsection{The FONDIL system}

The FONDIL system is responsible for the detection of similarities among meta-schemas/schemas within the OSIECS Kernel. In general, FONDIL provides inference services for Description Logic-based ontologies (Le Duc 2004). The expressiveness of such ontologies allows formalisation of the semantics of modelling languages (e.g UML, EXPRESS) and makes these semantics as explicit as possible. It is worth emphasising that formal and explicit semantics are crucial to automated deduction.

The FONDIL system is composed of three modules, namely ontology management, mediator, and inference engine. The heart of the FONDIL system is an inference engine that uses structural algorithms for non-standard inferences and optimised algorithms for standard inferences Le Duc, 2004; Lima et al. 2005b). FONDIL uses the inference engine to deduce new knowledge, using ontologies as the primary source of knowledge. The knowledge deduced is essentially new relations among the ontological concepts. FONDIL initially considers that the ontology manager needs some help to exploit all the possible relationships among the concepts within a single ontology. This help is even more necessary when considering several SRs that were (likely) developed independently from each other. The relationships among them (if they exist) are usually implicitly defined. These relationships can be viewed more as knowledge to be detected rather than knowledge to be predefined in the SRs. FONDIL's function within OSIECS is to assist in the refinement of the semantic mappings detected among the SRs (Lima et al. 2005b).

\subsection{Syntactic conversion and semantic analysis}

The mapping process involves three main aspects of SRs: the structures, the syntax, and the semantics. To solve syntax problems, the recommended solution is to represent the original SRs in neutral format; this can be achieved through conversion if necessary. The converted versions are then free of syntactical problems. Structural and semantic-related problems are solved through a semi-automatic process ( Lima et al. 2005a).

Before describing the process of creation of the OSIECS meta-model/model, it is worth noting that the meta-schemas used to form OSIECS were originally represented in different formalisms, as follows: EXPRESS is used in ISO 12006-3 and IFC, and UML is used in e-COGNOS and bcXML.

The Converter works with the metaschemas/schemas in their original formats and produces the corresponding OWL versions. The experts play a very strategic role in this phase, since they analyse the SRs' meta-schemas/schemas and create a set of conversion rules (written in Java) that allows conversion of each entity from their original format into OWL. This transformation must preserve the semantics of the converted entities. This set of rules is used by the $\mathrm{JavaCC}^{3}$ tool, which generates "transformers" capable of automatically translating any meta-schema/schemas written in the original format into OWL. During the OSIECS development, two "transformers" were generated to support the translations of both EXPRESS and UML to OWL.

\subsection{Detection of mappings}

As previously stated, the Detector of mappings uses the FONDIL inference engine to detect the similarities between each pair of concepts belonging to two different SRs. The similarity between two concepts is defined in four levels, according to its granularity, Let us consider two concepts $C 1$ and $C 2$ belonging to two meta-schemas. Firstly, the inference engine verifies if they are equivalent according to the OWL semantics. In case of equivalence, this result will be sent to the Validator.

\footnotetext{
${ }^{3} \mathrm{JavaCC}$ is available at https://javacc.dev.java.net
} 
Otherwise, those concepts are sent to the Subsumption Detection component that will check if one concept is subsumed by the other. If these concepts are not subsumed to each other, the similarity between them is evaluated by the Intersection Detection, LCS and Difference Detection components, which will deal with intersections, unions and differences among the concepts. This allows a more accurate detection of similarities between the two concepts. The similarities between the meta-schemas must be validated in order to produce the OSIECS metamodel.

\subsection{Matching the entities}

The similarities found in the previous stage are used over the schemas of the SRs, following a specialisation process. For instance, let $A$ be an entity from the e-COGNOS meta-schema and $B$ an entity from the ISO 12006-3 meta-schema. Thus, $S(A, B)$ represents a similarity between those entities. This similarity is then matched to the entities of the correspondent e-COGNOS and LexiCon schemas, $S^{\prime}(a, b)$. All the entities matched at the schema level of the selected SR compose the OSIECS model

\subsection{OSIECS meta-model and model}

Basically, the OSIECS meta-model and model are mapping tables that identify and establish the semantic correspondence between the entities forming the SRs. The OSIECS meta-model is the set of tables mapping the meta-schemas of the SRs forming OSIECS, while the OSIECS model is the set of tables mapping the schemas of SRs forming OSIECS. Both meta-model $/$ model were created during the FUNSIEC project and evaluated by the experts involved in the creation process.

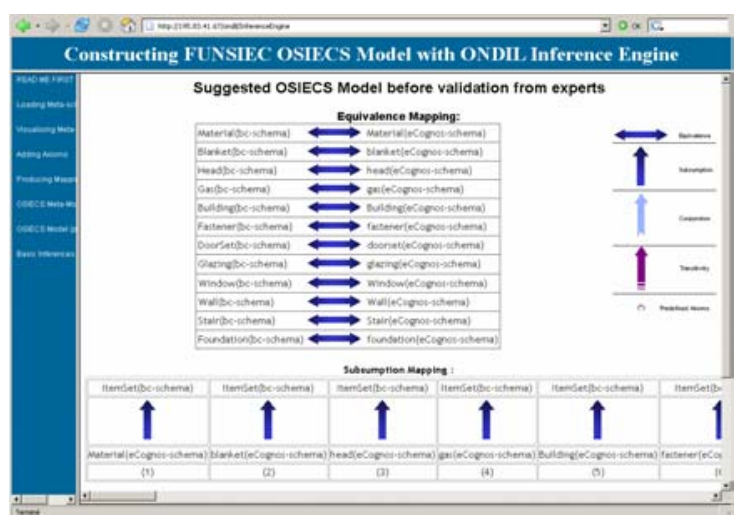

Figure 3. Partial view of the OSIEC model
Figure 3 shows a partial view of the OSIECS model illustrating equivalence mapping and subsumption mappings between bcXML and e-COGNOS.

\section{E-SERVICES PROVISION}

Although OSIECS primary purpose is to map construction related SRs, it also facilitates the provision of construction related e-services. Current software application-based collaboration requires integration through shared semantic resources. FUNSIEC argues that attention needs to be paid to direct support for business transactions and processes. This would enable organizations to migrate their legacy/commercial application systems, articulated around proprietary semantic resources, to higher order interoperable applications supporting real business processes (Rezgui and Meziane 2005).

The OSIECS Kernel can provide e-services over SRs for the construction sector. The initial list of OSIECS e-services concentrates on the enhancement of the OSIECS meta-model and model, including the following services: (i) Automatic conversion of SRs written in EXPRESS/UML (both meta-schemas and schemas) to OWL. (ii) Verification of the 'compatibility level' of a given SR (represented in EXPRESS/UML) regarding the OSIECS metamodel/model. (iii) Mapping report amongst SRs represented in OWL (Barresi et al. 2005).

Considering that OSIECS can be promoted and adopted, at least for the experimentation level, future e-services are to consider the creation and publication of e-catalogues, the creation and management of SRs (taxonomies and ontologies, in this case), and semantic mapping amongst SRs.

\section{CONCLUSION}

This paper presented part of the research carried out in the FUNSIEC project. By using the FUNSIEC methodology experts successfully developed the OSIECS Kernel, mapped four SRs between each other, and created the OSIECS meta-model and the OSIECS model. The FUNSIEC methodology is expected to be used in the future to integrate new SRs to the existing pool of resources already included in OSIECS. This will require new mappings and methods to be considered.

The FUNSIEC approach began with the characterisation of the domains, selection of 
pertinent SRs and the subsequent analysis of their meta-schemas/schemas. The SRs selected to form OSIECS, were converted into OWL. This process was performed semi-automatically by experts that extracted a set of conversion rules to feed the JavaCC, which created the respective transformers that were consequently used to perform the conversion of the SRs into OWL. The converted meta-schemas were semantically compared and mapped using the FONDIL system. The final output was the OSIECS meta-model. The OSIECS model was then produced using the same process.

Finally, another important aspect of the OSIECS Kernel is its ability to support a multitude of eservices. For the time being, the OSIECS Kernel provides e-services targeting the enrichment of the OSIECS meta-model/model. More services are expected to be included in this list in the near future.

\section{ACKNOWLEDGMENTS}

The authors would like to thank the members of the FUNSIEC consortium for their valuable contributions to the research as well as the financial support from the European Commission under the IST and eContent programs.

\section{REFERENCES}

Aussenac-Gilles, N., Biébow, B., and Szulman, S., 2001. Revisiting ontology design: a methodology based on corpus analysis. In 12th International Conference in Knowledge Engineering and Knowledge Management (EKAW'00), Lecture Notes in Artificial Iintelligence, vol. 1937, Berlin: Springer, , 396-408.

Barresi, S., Rezgui, Y., Lima, C., and Meziane, F., 2005. Architecture to support semantic resources interoperability. In Proceedings of the First International Workshop on Interoperability of Heterogeneous Information Systems, New York: ACM Press, 79-82

Bechhofer, S., Horrocks, I., Goble, C., and Stevens, R., 2001. OilEd: a reasonable ontology editor for the Semantic Web. In Joint German/Austrian conference on Artificial Intelligence (KI 01), Lecture Notes in Artificial Intelligence, vol. 2174, Berlin: Springer, 396-408.

Blazquez, M., Fernandez, M., Garcia-Pinar, J..M., and Gomez-Perez, A., 1998 Building ontologies at the knowledge level using the ontology design environment. In Proceeding of KAW 98 (Knowledge Acquisition Workshop), Banff, Canada, 18-23 April.

Chalupsky, H., 2000. OntoMorph: a translation system for symbolic knowledge, In Seventh International
Conference on Principles of Knowledge Representation and Reasoning (KR2000), San Francisco, Morgan Kaufmann, 471-482.

CoMMA, 2000. Corporate Memory Management through Agents. In Proceedings of E-Work \& E-Business, Madrid.

Corcho, O., Fernando-Lopez M., and Gomez-Perez A., 2003. Methodologies, tools and languages for building ontologies. Where is their meeting point? Data and Knowledge Engineering 46, 41-64.

eConstruct, 2001.Final edition of the $b c X M L$ Specifications [online]. Available from: http://www.econstruct.org/6public/bcxml_cd/publicdel iverables/d103_v2.pdf [Accessed 10 June 2005]

Euzenat, J., 1996. Corporative memory through cooperative creation of knowledge bases and hyperdocuments. In Proceedings of the 10th Knowledge Acquisition for Knowledge-Based Systems Workshop (KAW96), Banff.

Farquhar, A., Fikes, R., and Rice, J., 1996. The Ontolingua Server: A Tool for Collaborative Ontology Construction. In Proceedings of the 10th Knowledge Acquisition for Knowledge-Based Systems Workshop (KAW96), Banff, 44.1-44.19.

Fernandez-Lopez, M., 1999. Overview of methodologies for building ontologies. In Proceedings of the IJCAI99 workshop on ontologies and problem-solving methods (KRR5), Stockholm, August 2.

Grosso, E., Eriksson, H., Fergerson, R.W., Tu, S.W. and Musen, M. M., 1999. Knowledge modelling at the millennium - the design and evolution of Protégé2000. In Proc. the 12th International Workshop on Knowledge Acquisition, Modeling and Mangement (KAW'99), Banff.

Gruber, T., 1994. Towards principles for the design of ontologies used for knowledge sharing. International Journal of Human-Computer Studies (IJHCS), 43(5/6), 907-928.

Gruninger, M., and Fox, M.S., 1995. Methodology for the design and evaluation of ontologies. In Workshop on Basic Ontological Issues in Knowledge Sharing, Montreal.

Guarino, N., Masolo, C., and Vetere, G., 1999 OntoSeek: ContentBased Access to the Web. IEEE Intelligent Systems, Vol. 14, No. 3, May/June.

Guarino, N., and Welty, C., 2000. Ontological analysis of taxonomic relationships. In 19th International Conference on Conceptual Modeling (ER'00), Lecture Notes in Computer Science, vol. 1920, Berlin: Springer, 210-224.

Holsapple, C.W., and Joshi, K.D., 2002. A collaborative approach to ontology design. Communication of the ACM, 45 (2), 42-47.

ISO, 2004, ISO DIS 12006-3. Organisation of information about construction works. Part 3. Framework for object-oriented information. International Standards Organisation.

Kalfoglou, Y., and Robertson, D., 1999. Use of formal ontologies to support error checking in specifications. In Fensel, D., and Studer, R. (Eds.), 11th European 
Workshop on Knowledge Acquisition, Modeling and Management (EKAW'99), Lecture Notes in Artificial Intelligence, vol. 1621, Berlin: Springer, 207-224.

Kalfoglou, Y., and Schorelmmer, M., 2003. Ontology mapping: the state of the art. The Knowledge Engineering Review, v.18, no.1, 1-32.

Kayed, A., and Colomb, R.M., 2002. Extracting ontological concepts for tendering conceptual structures. Data and Knowledge Engineerin,g, 40, 7189.

Kietz, J.U., Maedche, A., and Volz, R., 2000. A method for semi-automatic ontology acquisition from a corporate intranet. In EKAW'00 Workshop on Ontologies and Texts, Juan-Les-Pins: CEUR Proceedings, vol. 51.

Klein, M., 2001 Combining and relating ontologies: an analysis of problems and solutions, In Gomez-Perez, A., Gruninger, M., Stuckenschmidt, H., and Uschold M. (Eds.), Workshop on Ontologies and Information Sharing, IJCAI'01, Seattle, USA, 4-5 August.

Klein, M., and Fensel, D., 2001. Ontology versioning on the Semantic Web, In First International Semantic Web Workshop (SWWS01), Stanford.

Klein, M., Fensel, D., Kiryakov, A., and Ognyanov, D., 2002. Ontology versioning and change detection on the Web. In Gomez-Perez, A., and Benjamins V.R. (Eds.), 13th International Conference on Knowledge Engineering and Knowledge Management (EKAW02), Lecture Notes in Artificial Intelligence, vol. 2473, Berlin: Springer.

Le Duc, C., 2004. Transformation d'ontologies basées sur la Logique de Description - Application dans le Commerce Electronique. Thesis (PhD), Université de Nice.

Lenat, D.B., and Guha, R.V., 1990. Building Large Knowledge-Based Systems: Representation and Inference. In the Cyc Project. Boston: AddisonWesley.

Li, W., and Clifton, C., 1994. Semantic integration in heterogeneous databases using neural network. In Proceedings of the 20th International Conference On Very Large Data Bases, San Francisco: Morgan Kaufmann Publishers, 1-12.

Lima, C., Ferreira-da-Silva, C., Le Duc, C., and Zarli, A., 2005a. A Framework to Support Interoperability among Semantic Resources. In Proceedings of INTEROP-ESA 2005, Geneva, Switzerland, February.

Lima, C., Ferreira da Silva, C., Sousa, P., Pimentão, J. P., and Le-Duc, C., 2005b. Interoperability among Semantic Resources in Construction: Is it Feasible?, CIB-W78 Conference, Dresden, Germany, July.

Lima, C., Storer, G., Zarli, A., and Ferreira-da-Silva, C., 2005c. Towards a Framework for managing standardsbased semantic e-resources in the European Construction Industry. In Proceedings of Construction Research Congress (CRC), San Diego, US, April.

McGuiness, D.L., Fikes, R., Rice, J., and Wilder, S., 2000 An environment for merging and testing large ontologies. In Seventh International Conference on Principles of Knowledge Representation and
Reasoning 5KR 2000, Breckenridge, USA, 12-15 April.

Milo, T., and Zohar, S., 1998. Using schema matching to simplify heterogeneous data transaction. In Proceedings of the 24th International Conference On Very Large Data Bases, San Francisco: Morgan Kaufmann Publishers, 122-133.

Neches, R., Fikes, R.E., Finin, T., Gruber, T.R., Senator, T., and Swartout, W.R., 1991. Enabling Technology for Knowledge Sharing. AI Magazine 12 (3), 36-56.

Noy, N.F., and Musen, M.A., 2000. PROMPT: Algorithm and Tool for Automated Ontology Merging and Alignment. In 17th National Conference on Artificial Intelligence (AAAI'O0), Austin.

Noy, N.F., and Klein, M., 2002. Ontology Evolution: Not the Same as Schema Evolution. Technical Report SMI-2002-0926, Stanford.

Partridge, C., 2002. The Role of Ontology in Integrating Semantically Heterogeneous Databases, Technical Report 05/02, LADSEB-CNR, Padova, Italy, June.

Pinto, H.S., Gomez-Perez, A., and Martins, J.P., 1999. Some issues on ontology integration. In Proceedings of the IJCAI-99 Workshop on Ontologies and Problem Solving Methods (KRR5), Stockholm, Sweden, 2 August.

Pinto, H.S., and Martins, J.P., 2000. Reusing ontologies. In Proceedings of AAAI 2000 Spring Symposium Series, Workshop on Bringing Knowledge to Business Processes, SS-00-03, AAAI Press, 77-84.

Rahm, E., and Bernstein, P.A., 2001. A Survey of Approaches to Automatic Schema Matching. VLDB Journal, Vol. 10, No. 4, Dec.

Rezgui, Y., 2001. Review of information and knowledge management practices state of the art in the construction industry, The Knowledge Engineering Review Journal, 16(3), 241-254.

Rezgui, Y., and Meziane, F., 2005. A web service implementation of a user centred knowledge management platform, International Journal of Intelligent Information Technologies, Vol. 1, No 4 Idea Group Publishing.

Stumme, G., and Ad Maedche, A., 2001. Ontology merging for federated ontologies on the semantic web. In Gomez-Perez, A., Gruninger, M., Stuckenschmidt, H., and Uschold, M. (Eds.), Workshop on Ontologies and Information Sharing, IJCAI'01, Seattle, USA, 4-5 August.

Sure, Y., Erdmann, M., Angele, J., Staab, S., Studer, R., and Wenke, D., 2002. OntoEdit: collaborative ontology engineering for the semantic web. In First International Semantic Web Conference (ISWC'02), Lecture Notes in Computer Science, vol. 2342, Berlin: Springer, 221-235. 